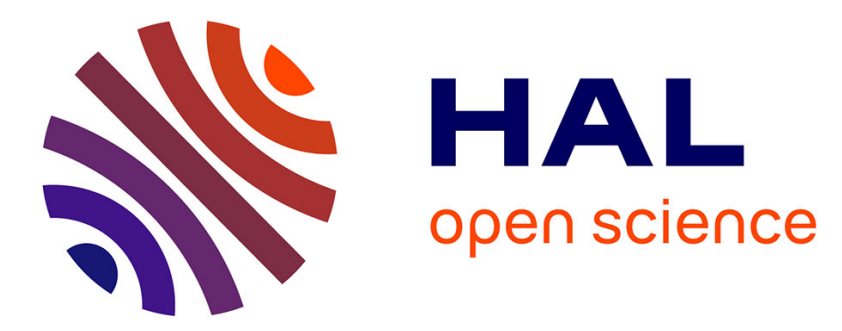

\title{
Molecular Doping of 2D Indium Selenide for Ultrahigh Performance and Low-Power Consumption Broadband Photodetectors
}

Ye Wang, Hanlin Wang, Sai Manoj Gali, Nicholas Turetta, Yifan Yao, Can Wang, Yusheng Chen, David Beljonne, Paolo Samorì

\section{To cite this version:}

Ye Wang, Hanlin Wang, Sai Manoj Gali, Nicholas Turetta, Yifan Yao, et al.. Molecular Doping of 2D Indium Selenide for Ultrahigh Performance and Low-Power Consumption Broadband Photodetectors. Advanced Functional Materials, 2021, 31 (30), pp.2103353. 10.1002/adfm.202103353 . hal-03321054

\section{HAL Id: hal-03321054 https://hal.science/hal-03321054}

Submitted on 16 Aug 2021

HAL is a multi-disciplinary open access archive for the deposit and dissemination of scientific research documents, whether they are published or not. The documents may come from teaching and research institutions in France or abroad, or from public or private research centers.
L'archive ouverte pluridisciplinaire HAL, est destinée au dépôt et à la diffusion de documents scientifiques de niveau recherche, publiés ou non, émanant des établissements d'enseignement et de recherche français ou étrangers, des laboratoires publics ou privés. 


\section{Molecular doping of two-dimensional indium selenide for ultrahigh performance and low-power consumption broadband photodetectors}

Ye Wang, Hanlin Wang, Sai Manoj Gali, Nicholas Turetta, Yifan Yao, Can Wang, Yusheng Chen, David Beljonne, Paolo Samori*

${ }^{1}$ University of Strasbourg, CNRS, ISIS UMR 7006, 8 Alleé Gaspard Monge, F-67000 Strasbourg, France.

E-mail: samori@unistra.fr

${ }^{2}$ Laboratory for Chemistry of Novel Materials, Université de Mons, Place du Parc 20, 7000 Mons, Belgium.

Keywords: Two-dimensional material, indium selenide, black phosphorus, doping, photodetectors, van der Waals heterostructures, p-n junctions 


\section{ABSTRACT}

Two-dimensional (2D) photodetecting materials have shown superior performances over traditional materials (e.g. silicon, perylenes) which demonstrated low responsivity (R) $\left(<1 \mathrm{AW}^{-1}\right)$, external quantum efficiency (EQE) $(<100 \%)$ and limited detection bandwidth. Recently, 2D indium selenide (InSe) stands out as high-performance field-effect transistors and photodetectors, yet via expensive and complex techniques. Here we show for the first time how molecular functionalization with a common surfactant molecule (didodecyldimethylammonium bromide) (DDAB) represents a powerful strategy to boost the (opto)electronic performances of InSe yielding major performance enhancements in phototransistor, Schottky junction and vdW heterostructures by a lithography-compatible fabrication route. The functionalization could controllably dope and heal vacancies in InSe, resulting in ultrahigh field-effect mobility $\left(10^{3} \mathrm{~cm}^{2} / \mathrm{Vs}\right)$ and photoresponsivity $\left(10^{6} \mathrm{~A} / \mathrm{W}\right)$, breaking the record of non-graphene-contacted 2D photodetectors. Our strategy towards the molecular doping of 2D photodetecting materials is efficient, practical, up-scalable and operable with ultra-low power input, ultimately paving the way to next-generation $2 \mathrm{D}$ opto-electronics. 


\section{MAIN}

Photodetectors capable of sensing light from ultraviolet (UV) to infrared (IR) have become key devices in a broad range of technologies comprising optical sensing, image recognition, motion detection, remote control, biomedical imaging, etc. ${ }^{[1-4]}$ Two-dimensional (2D) materials have been extensively studied during the last decade as promising photodetecting materials owing to their fast response, high responsivity, photodetectivity and broad wavelength detection. The wide selection of materials with tunable bandgap obtained by altering the layer numbers and the simple formation of van der Waals (vdW) heterostructures enabled to reach high responsivity $\left(10^{6} \sim 10^{7} \mathrm{AW}^{-1}\right)$, high detectivity $\left(D^{*}\right)\left(10^{10} \sim 10^{13} \mathrm{~J}\right.$ ones) and ultrafast photoresponse (on the $\mu$ s timescale). ${ }^{[1,5-12]}$ However, the operation of these high-performance devices requires high bias voltage yielding large power consumption. Such an issue represents a strong handicap for various technological applications such as photosensor in extreme environment, bio-medical imaging, portable devices, etc. 2D InSe has recently attracted a great attention because of its ultrasensitive photodetection characteristics outperforming common $2 \mathrm{D}$ semiconducting materials such as $\mathrm{MoS}_{2}$ and $\mathrm{WSe}_{2 .}{ }^{[5,6,8,13-16]}$ Such high performances was achieved by means of complex and specific techniques such as ion implantation and nanopatterning, which unfortunately drastically increase the fabrication costs. ${ }^{[6,7,17]}$ A powerful route for tuning physical and chemical properties of $2 \mathrm{D}$ materials, which has been thoroughly applied to graphene, TMDs and BP, consists in molecular functionalization via covalent and non-covalent strategies, resulting in doping, defect healing, increase in bio-compatibility, etc. $[18,19]$ Surprisingly, such approach has not yet been attempted with InSe for obtaining high-performance devices. Moreover, despite the high electron mobility of InSe, little effort has been devoted in combining InSe with other 2D materials to generate highly responsive $\mathrm{p}-\mathrm{n}$ photodetectors. This urges us to find viable strategies for the construction of high-performance photodetectors based on InSe and InSe-based p-n junctions.

Here we show how the functionalization with a common surfactant molecule represents a powerful strategy to boost the (opto)electronic performances of 10-15 nm thick InSe flakes exfoliated from commercial crystals yielding to major property enhancements in InSe based phototransistor, lateral Schottky junction and BP-InSe vdW p-n heterostructures. For the first 
time we have also fabricated high-responsivity, fast response, low power input 2D phototransistors through a lithography-compatible route in which the performances are enhanced via the functionalization with organic molecules.

\section{Dielectric engineering InSe field-effect transistor}

In order to cast light onto the role of the dielectric characteristics of the substrate, as-exfoliated flakes were transferred onto both $\mathrm{Si} / \mathrm{SiO}_{2}$ and polymer-coated $\mathrm{Si} / \mathrm{SiO}_{2}$ substrate. Figure 1a displays the transfer $\left(\mathrm{V}_{\mathrm{g}}-\mathrm{I}_{\mathrm{ds}}\right)$ curve of InSe on $\mathrm{SiO}_{2}$. It reveals modest transport performances with electron mobilities around $0.01 \mathrm{~cm}^{2} / \mathrm{Vs}$, being considerably lower than other 2D semiconductors. Such low performances can be ascribed to the abundance of charge traps at the $\mathrm{InSe} / \mathrm{SiO}_{2}$ interface which is detrimental to photodetection, also limiting the use of as-prepared InSe as a platform for molecular functionalization, because the leakage current would have a similar magnitude of the drain-source current, yielding device instability. ${ }^{[20,21]}$ Therefore, it is imperative to improve the local environment where InSe is embedded, and in particular its dielectric characteristics. Towards this end, divinyltetramethyl disiloxanebis(benzocyclobutene) (BCB) was chosen as trap-free dielectric polymer for its easy processability, high thermal and chemical resistance, being an optimal choice for microfabrication of 2D materials. ${ }^{[22]}$ The precursor-free solution-processable and lithography-friendly characteristics made $\mathrm{BCB}$ much more advantageous compared to other trap-free dielectrics such as poly(methyl methacrylate) (PMMA, highly soluble in common solvents), polyimide (PI, usually precursor-involved) and hexagonal boron nitride (h-BN, very costly for both CVD-grown and exfoliated materials). The hysteresis of transfer curves have been largely decreased by $30 \mathrm{~V}$ compared to pure $\mathrm{SiO}_{2}$ supported devices (Figure S1a, Supporting Information). The top-contact device architecture is depicted in Figure 1b. The resulting capacitance of the in-series capacitor drops from $12.7 \mathrm{nF} / \mathrm{cm}^{2}$ for $\mathrm{SiO}_{2}$ to 6.05 $\mathrm{nF} / \mathrm{cm}^{2}$ for BCB. As shown in Figure 1a, the 2D conductivity $(\sigma)$ of InSe drastically increases in devices from $(1.99 \pm 0.966) \times 10^{-4} \mu \mathrm{S}$ on $\mathrm{SiO}_{2}$ to $25.30 \pm 11.19 \mu \mathrm{S}$ on $\mathrm{BCB}$, with turn-on voltage fixed around $0 \mathrm{~V}$, thereby indicating the unchanged degree of doping on different dielectrics. Similarly, the field-effect mobility of InSe undergoes a major increase 
from a max value of $0.0116 \mathrm{~cm}^{2} / \mathrm{Vs}$ on $\mathrm{SiO}_{2}$ to $688.2 \mathrm{~cm}^{2} / \mathrm{Vs}$ on $\mathrm{BCB}$. The mechanism could be explained through Figure 1c where large defect densities (e.g. point defect $\mathrm{P}_{\mathrm{b}}$ center) are formed during the thermal oxidation of silicon, in addition to the polarized surface optical (SO) phonon modes in Si-O bond oscillatory motions, limiting the mobility of electron transport by introducing localized states and participating in Coulomb scattering. ${ }^{[23-26]}$ By using $\mathrm{BCB}$ as a gate dielectric, the interface trap density $D_{i t}$ between InSe and the dielectric has decreased by one order of magnitude, leading to a more effective screening of Coulomb scattering, thus increasing the charge carrier mobility and channel conductance.

\section{Ionic molecular doping of InSe field effect transistor}

Although the use of BCB dielectric largely improves the channel conductivity of InSe, the device is in OFF state at $\mathrm{V}_{\mathrm{g}}=0 \mathrm{~V}$. This raises a major concern in operating the photodetector with low power and the photocurrent value would be limited by the unfavourable charge injection from the metal contact. This problem can be overcome by lowering the Schottky barrier via doping. For 2D materials, molecular doping has become a most viable approach to tune the Fermi level of semiconducting materials thanks to the large surface-to-volume ratio for physi(chemi)sorption. ${ }^{[27-30]}$ Instead of employing the most common doping strategies of 2D materials based on aromatic molecules, we have focussed our attention to a well-established surfactant molecule, didodecyldimethylammonium bromide (DDAB), containing ionic moiety that can effectively interact with the surface of InSe. Transfer curves of InSe FET were measured after spin-coating the solution in TCE from $10^{-6}$ $\mathrm{g} / \mathrm{L}$ to $1 \mathrm{~g} / \mathrm{L}$ concentrations. Figure 1d shows that the threshold voltage $\left(\mathrm{V}_{\text {th }}\right)$ shifts negatively with increasing concentrations (output curve changes displayed in Figure S1b and Figure S1c, Supporting Information). The maximum $\Delta V_{t h}$ obtained within 25 devices with the highest concentration amounts to $42.58 \mathrm{~V}$, corresponding to $1.7 \times 10^{12} / \mathrm{cm}^{2}$ of charge carrier density change. We exclude possible doping from the solvent after thermal annealing, which is ruled out in by means of the control experiment reported in Figure S2 in Supporting Information.

For 2D materials, molecular doping has become a most viable approach to tune the Fermi level of semiconducting materials thanks to the large surface-to-volume ratio, high 
chemical reactivity and sensitivity. The choice of molecules from the huge library of molecules to functionalize with specific $2 \mathrm{D}$ material is crucial. ${ }^{[30,31]}$ Fermi level shift in 2D materials could be monitored by Kelvin probe force microscopy: Figure 1e shows the surface potential image of a $5.5 \mathrm{~nm}$ thick InSe flake before and after its functionalization with DDAB. The profile in Figure 1f reveals that the molecular functionalization determines an increase in surface potential of $200 \mathrm{meV}$. The resulting work function $\varphi$ obtained in InSe flake region calibrated with respect to $\varphi_{\mathrm{Pt} / \mathrm{Ir}}$ shows a decrease from 4.9 to $4.7 \mathrm{eV}$, indicating the Fermi level lifting towards the conduction band of InSe (Figure S3 in Supporting Information). Furthermore, Figure1e provides evidence for a uniform magnitude in surface potential across the flake, denoting a homogeneous modulation of electronic properties of InSe upon DDAB functionalization.

To fully interpret the origin of such strong doping induced by simple ionic surfactant such as DDAB, Density Functional Theory (DFT) calculations were performed to model the hybrid InSe/DDAB system. Since defects (e.g. Se vacancies) could be generated both during the synthesis of InSe crystal and the delamination by mechanical exfoliation, we considered InSe single layers in either the pristine form (dubbed 'InSe' in the following) or including 3\% Se vacancies (InSe-3\%SeV) (Figure S4). We explored two molecular conformations for adsorption, with conformation 1 (denoted as InSe $(-3 \% \mathrm{SeV}) / \mathrm{DDAB}-1)$ and conformation 2 (denoted as InSe $(-3 \% \mathrm{SeV}) / \mathrm{DDAB}-2)$ corresponding to the $\mathrm{Br}$ ion being far and close to the InSe surface, respectively (Figure 1g and Figure S5-8 in Supporting Information). The resulting calculated work functions are listed in Figure1h. We find that functionalization with DDAB molecules determines a work function decrease by $0.09 \mathrm{eV}$ (pristine InSe) and 0.13 $\mathrm{eV}$ (defective InSe) in conformation 1, while the corresponding values in conformation 2 are $0.43 \mathrm{eV}$ (pristine InSe) and $0.53 \mathrm{eV}$ (defective InSe). The shifts in work function calculated for the two conformations are in line with the experimental values and result from the combined effect of a partial charge transfer from the molecule to the surface (n-doping) together with a dipolar contribution (of obviously opposite signs in the two conformations considered). Furthermore, we have also observed a systematic improvement of field-effect mobility up to $2785 \mathrm{~cm}^{2} / \mathrm{Vs}$ upon the DDAB functionalization, which is due to the combined 
effect of molecular encapsulation and lowering of contact resistance, as detailed in Supporting note 2 in Supporting Information. ${ }^{[32]}$

Alongside the DDAB effect on the electrical properties of InSe, we also observed major changes in the optical properties. While Raman modes of InSe have not revealed major changes upon functionalization with $\mathrm{DDAB}$, indicating that the molecular functionalization does not modify the crystal structure of the $2 \mathrm{D}$ material, a strong quenching and a $0.8 \mathrm{eV}$ redshift in the photoluminescence (PL) spectra have been monitored (Figure S12 in Supporting Information). The variations in PL are in line with previous observations in other n-type 2D materials, such as $\mathrm{MoS}_{2} \cdot{ }^{[33]}$

\section{Molecular functionalized InSe field-effect phototransistor (photoFET)}

Phototransistors are one among the most investigated device structures for 2D photodetectors. Their architectures are identical to bottom-gate top-contact FET, as displayed in Figure 1b. Figure 2a-c shows the gate-dependent photoresponse of $\mathrm{InSe}$ on $\mathrm{SiO}_{2}, \mathrm{BCB}$ and InSe/DDAB on BCB. A prominent selective photodetection of UV light (365 nm) is observed for both $\mathrm{InSe}$ on $\mathrm{SiO}_{2}$ and $\mathrm{BCB}$. For phototransistor on $\mathrm{SiO}_{2}$, even at highly gated region, the measured photocurrent is only in sub-microampere scale with $\mathrm{I}_{\mathrm{on}} / \mathrm{I}_{\mathrm{off}}$ around $10^{3}$. The modification of the dielectric layer with BCB leads to an efficient suppression of the undesired recombination from charge traps located at InSe/dielectric interface, thus enhancing the $\mathrm{I}_{\mathrm{on}} / \mathrm{I}_{\mathrm{off}}$ ratio up to $10^{6}$ (Figure $\mathbf{2 b}$ ). The photoresponse rise /decay time has also significantly shortened from $288 / 447 \mathrm{~ms}$ on $\mathrm{SiO}_{2}$ to $17.33 / 16.76 \mathrm{~ms}$ on $\mathrm{BCB}$. Nevertheless, the photoresponse is still limited by the existence of Se vacancies which could act as traps for photo-generated charge carriers. To minimize such effect, we functionalized the InSe channel with DDAB, by exploiting the propensity of the latter compound to stably adsorb on the Se vacancy sites. The healing of Se vacancies by DDAB could help to restore the crystal structure thereby suppressing the recombination of the photo-generated charges in vacancy traps. Figure $2 \mathbf{c}$ provides distinct evidence that such functionalization yields a higher photoresponse. Such enhancement can be ascribed not only to the contribution of Se vacancy healing, but also to the molecular doping induced shift of the $\mathrm{V}_{\text {th }}$ in InSe FET drawing the device to ON state at $\mathrm{V}_{\mathrm{g}}=0 \mathrm{~V}$ which would otherwise be realized by applying a large electrical gate up to $80 \mathrm{~V} \cdot{ }^{[1,8,13,34]}$ Therefore, with zero 
contribution from the gate bias, the barrier from the contact is low enough for the photocurrent to tunnel through effectively (Figure 2d). Combining these two factors, we have obtained a reasonably high photocurrent of $12 \mu \mathrm{A}$ by simply applying $1 \mathrm{~V}$ of bias voltage in total. The device performance is proved to be stable within light pulse cycles and reproducible among different devices (Figure S13,14, 29 in Supporting Information).

Responsivity (R) of incident wavelength $\lambda$ is a key parameter to evaluate photocurrent generation of photodetectors. The spectral photoresponse of incident light from $300 \mathrm{~nm}$ to $690 \mathrm{~nm}$ for $\mathrm{InSe}$ on $\mathrm{SiO}_{2}$, InSe on $\mathrm{BCB}$ and $\mathrm{InSe} / \mathrm{DDAB}$ in Figure 2e reveals a single photodetective band from $300 \mathrm{~nm}$ to $400 \mathrm{~nm}$. An exponential enhancement of $10^{6}$ from $\mathrm{SiO}_{2}$ to $\mathrm{BCB}$ as dielectric is observed. Upon functionalization with $\mathrm{DDAB}, \mathrm{R}$ reaches $10^{5} \mathrm{~A} / \mathrm{W}$ (Figure S 15d). Figure 2f and $\mathbf{g}$ portrays the photoresponsive characteristics with respect to incident light power intensity $(P)$ of $365 \mathrm{~nm}$ for InSe and InSe/DDAB. The photocurrent was found to scale linearity with light power density, complying $I_{p h} \propto P^{\alpha}$. The linearity factor $\alpha$ is calculated to be 0.448 for InSe and 0.658 for InSe/DDAB, implying a reduction of traps. ${ }^{[35]}$ The time-dependent photoresponse at variable power density is displayed in Figure $\mathbf{S} 16$. EQE represents the efficiency of charge carrier collected per single absorbed photon. In pristine InSe devices, the $\mathrm{R}$ and EQE in the low power region $\left(5 \mu \mathrm{W} / \mathrm{cm}^{2}\right)$ have reached values of $2 \times 10^{5} \mathrm{~A} / \mathrm{W}$ and $7 \times 10^{7} \%$, respectively, indicating an ultra-sensitive photodetection for low-power light. The DDAB functionalization enhances $\mathrm{R}$ up to $1 \times 10^{6} \mathrm{~A} / \mathrm{W}$ and $\mathrm{EQE}$ to $5 \times 10^{8} \%$. Moreover, the photoconductive gain $(\mathrm{G})$, which aid to understand the photogating effect, is evaluated with details reported in Supporting note 3 in Supporting Information. Finally, $D^{*}$ quantifies the signal to noise ratio of a given photodetection area. Power dependent detectivity of InSe and InSe/DDAB is plotted in Figure $2 \mathrm{~g}$ ranging from $10^{9}$ to $10^{12}$ for InSe. After functionalization with DDAB, the highest detectivity values reach $\sim 10^{13}$ Jones for $5 \mu \mathrm{W}$ irradiations. Overall, compared to previously reported 2D photodetectors, our molecularly functionalized phototransistors operating with ultra-low voltages $\left(\mathrm{V}_{\mathrm{g}}=0 \mathrm{~V}\right.$, $\mathrm{V}_{\mathrm{ds}}=1 \mathrm{~V}$ ) have displayed extremely high responsivity up to $10^{6} \mathrm{~A} / \mathrm{W}, \mathrm{EQE}$ approaching $10^{8} \%$, and detectivity of $10^{13}$ Jones in the 300 to $690 \mathrm{~nm}$ wavelength region. It also exhibits ultra-fast time response for low-power $\left(50 \mu \mathrm{W} / \mathrm{cm}^{2}\right.$, Figure $\mathbf{S 1 7}$ in Supporting Information) illumination reaching a response time of $4.9 \mathrm{~ms}$. 


\section{Molecular functionalized InSe asymmetric Schottky junction}

Photodetectors based on 2D lateral p-n junctions have been realized by either controlling the semiconducting channel region by selective doping, or by manipulating the electron/hole injection through the use of asymmetric metal contact. ${ }^{[22,36-40]}$ Here we adopt both strategies to realize high performance lateral p-n junction based on multi-layer InSe by chemical doping with DDAB. The device structure is shown in Figure 3a. We have carefully chosen metals with high and low work functions (Pd: $\sim 5.6 \mathrm{eV} \mathrm{Cr:} 4.4 \mathrm{eV}$ ) to form large Schottky barrier difference. ${ }^{[41-43]}$ The metal-semiconductor contact is analysed in Figure S18 in Supporting Information, revealing large Schottky barrier with Pd and smaller Schottky barrier on Cr. Therefore, a depletion region is formed at the Pd-InSe interface thereby p-doping the contact region of InSe (Figure 3b). While the n-doping by DDAB is uniform for InSe, the hole transport region is protected by few-layer of hexagonal boron nitride (h-BN) as displayed in the AFM image in Figure 3c. The device showed a gate-dependent rectification where the rectification ratio amounts to 198 at $\mathrm{V}_{\mathrm{g}}=0 \mathrm{~V}$ in dark (Figure 3d). After doping with DDAB, the reverse bias current maintained in sub-nanoampere range with a 5-fold increase in the forward bias current, reaching a rectification ratio of 716 . The obtained functionalized Schottky junction exhibits an ideal factor $\eta$ around 1 , rendering it an ideal diode (Supporting note 4 in Supporting Information). The wavelength-dependent photodetective properties of such a p-n junction are evaluated in Figure $\mathbf{3 e}$ and $\mathbf{f}$. Similar to InSe phototransistor, the lateral p-n junction shows selective photoresponse for UV light (Figure S20 in Supporting Information). Additional photodetection test on $850 \mathrm{~nm}$ and $940 \mathrm{~nm}$ near infrared (NIR) light is presented in Figure S21 in Supporting Information. The device displays strong power dependence (Figure 3g-i and Figure S22 in Supporting Information). The linearity factor drops from 0.8440 for InSe/h-BN to 0.8065 for InSe/h-BN/DDAB, likely because of the inhomogeneity in the channel where the n-region is governed by the physisorbed organic molecules, while the p-region is screened by crystalline inorganic h-BN. Furthermore, by calculating R, EQE and $\mathrm{D}^{*}$, the lateral P-N junction reaches high R and EQE exceeding $10^{3} \mathrm{~A} / \mathrm{W}$ and $3 \times 10^{5} \%$ upon $5 \mu \mathrm{W} / \mathrm{cm}^{2}$ illumination after DDAB functionalization, being 4 times greater than undoped junction. Simultaneously, $D^{*}$ also shows 10 -fold 
enhancement at low power illumination, reaching $4 \times 10^{11}$ Jones. The photoresponse time of the lateral P-N junction is also found to be ultrafast for both unfunctionalized and functionalized samples, which all decreased below $1 \mathrm{~ms}$ (Figure S23 in Supporting Information). Compared to previously reported InSe lateral P-N junctions, our molecular functionalized device not only represents a novel device architecture that is highly suitable for exploring the photodetection of InSe, but also displayed record performance when operating at very low voltage inputs, demonstrating the power of molecular doping in InSe Schottky junctions.

\section{Molecular functionalized BP-InSe van der Waals p-n heterostructures}

Two-dimensional materials have been widely exploited as building blocks for vdW p-n heterostructures with tuneable bandgaps by varying the material composition and thicknesses. As an n-type semiconducting material, InSe could form type-II band alignment with various p-type 2D semiconductors including the archetypical natural p-doped 2D material is black phosphorus (BP), which possesses a small bandgap of $\sim 0.3 \mathrm{eV}$. The band alignment of BP and InSe is demonstrated in Figure S24 in Supporting Information. While the development of functional devices based on BP heterostructures with graphene, $\mathrm{MoS}_{2}, \mathrm{ReS}_{2}$, etc. have been widely reported in the literature, only two recent papers reported BP-based heterostructure with InSe which unfortunately did not demonstrate reliable high-performance photodetectors as other $2 \mathrm{D}$ materials. ${ }^{[44-48]}$ This is achieved here, where we first focus on the dielectric engineering of the BP-InSe heterostructure showing evident performance enhancement of the P-N junction, as discussed in detail in Supporting note 5 and Figure S25-26 in Supporting Information.

Based on previous discussions, electron doping in the n-region is beneficial for enhancing the performances of $\mathrm{P}-\mathrm{N}$ junctions. Therefore, it is reasonable to envisage a strong molecular n-dopant such as DDAB could easily promote the photodetection properties. In order to isolate BP from molecular doping, we partially passivated the BP flake with h-BN to prevent its exposure to molecules, as displayed in Figure 4a. After functionalization with DDAB, a nearly 10 -fold increase in the forward bias photocurrent has been recorded, while the reverse bias current retained the same magnitude (Figure 4b-c, Figure S27 in Supporting 
Information). Such observations can be explained by the upshift of Fermi level in the n-InSe region, prompting a larger built-in potential in the depletion region. This allows photoinduced excitons to easily dissociate into photoelectrons (holes) across the heterostructure, assisted by an external drain bias of $1 \mathrm{~V}$. In this regard, the photoresponse time has also drastically decreased from $24.40 / 36.41 \mathrm{~ms}$ to $0.96 / 2.97 \mathrm{~ms}$, which could be ascribed to a drifting of photocarriers (electrons to $\mathrm{n}$-InSe and holes to $\mathrm{p}$-BP) facilitated by the larger potential difference at the vdW interface as well as the molecular functionalization filling the defect states of InSe, thereby reducing the scattering of photocarriers that would notably slow down the photoresponse time (Figure 4d-e). The enhancement of the functionalization is proved to be reproducible in different devices (Figure S28 in Supporting Information). Compared to pure InSe with single absorption band (Figure 4f), the spectral $\mathrm{R}$ of the heterostructure showed two additional absorption bands from 450 to $550 \mathrm{~nm}$ and from 600 to $690 \mathrm{~nm}$. It is attributed to the presence of BP who possesses a much smaller bandgap, is able to generate larger photocurrent at the large wavelength region compared to pure InSe, contributing to the total photocurrent response, which is reflected as additional photocurrent absorption bands in the spectrum. The $\mathrm{R}$ value reaches a maximum value of $46 \mathrm{~A} / \mathrm{W}$ and $537 \mathrm{~A} / \mathrm{W}$ at $365 \mathrm{~nm}$ before and after the molecular functionalization, respectively. The ultrahigh responsivity reaches a record value among reported 2D-2D P-N heterostructures, especially, by operating the device with only $1 \mathrm{~V}$ of voltage input. The power-dependent photodetection from $5 \mu \mathrm{W} / \mathrm{cm}^{2}$ to $4120 \mu \mathrm{W} / \mathrm{cm}^{2}$ before and after the DDAB doping in Figure $4 \mathbf{g}$ to $\mathbf{i}$ indicates the photocurrent improvement to be universal for different power density. By calculating the linearity factor, we obtain $\alpha=0.44$ for non-functionalized and $\alpha=0.48$ for functionalized heterostructure, revealing a reduction of the amount of impurities (e.g. defects) in the P-N junction. The highest $\mathrm{R}$ and $\mathrm{EQE}$ exceeded $10^{3} \mathrm{~A} / \mathrm{W}$ and $3.5 \times 10^{5} \%$ after the functionalization, being almost 2 orders of magnitude larger than the unfunctionalized device while $D^{*}$ remains on the same range of $10^{11}$ Jones. The successful realization of vdW BP-InSe P-N heterostructure and its performance improvement provides even stronger evidence of the high relevance of molecular functionalization of InSe to boost performances in a broad range of opto-electronic device types, and in particular for low-power ultra-responsive photodetectors. 


\section{CONCLUSIONS}

In summary, we have demonstrated novel strategies for markedly improve the performances of multifunctional opto-electronic devices based on few-layer InSe by means of dielectric engineering using trap-free polymer and molecular functionalization with DDAB. By combining experimental work with theoretical calculations, we showed that DDAB could form a stable physisorbed layer onto the surface of InSe by lowering the Fermi level and, at the same time, healing the defect states of InSe. The resulting transistors displayed field-effect mobilities exceeding $10^{3} \mathrm{~cm}^{2} / \mathrm{Vs}$ with a high doping density of $10^{12} / \mathrm{cm}^{2}$. By further exploring molecular functionalized InSe as photodetectors, we successfully fabricated InSe devices in the form of phototransistor, Schottky contact lateral P-N junction and vdW vertical P-N junction with ultra-high performances even when operated at very low bias input. The phototransisitor reaches ultra-high photoresponsivity of $10^{6} \mathrm{~A} / \mathrm{W}$, meanwhile showing very fast photoresponse below $5 \mathrm{~ms}$. The photoresponse time was efficiently decreased to sub-microsecond scale with molecular doped P-N junction, meanwhile the EQE was enhanced up to $10^{5} \%$, thus outperforming previous reports of 2D P-N junctions (Supporting

Table 2 in Supporting Information). The high photoresponsivity is reproducible among devices (Figure S29 in Supporting Information). This work provides distinct evidence of the full potential of molecular doping of InSe as viable platform for improving the functional complexity and ultimately fabricate high-performance 2D opto-electronic device. More generally, the employed chemical functionalization does not require sophisticated instruments; it is efficient, practical, up-scalable, and widely applicable to manipulate at will various physical properties of 2D semiconductors for large-area applications.

\section{METHODS}

Sample preparation. BCB dielectric was prepared by diluting Cyclotene 3022-46 (Dow Chemistry) is to $20 \%$ vol with mesitylene. The solution is spin-coated onto thermally oxidized heavily $n$-doped silicon substrates (Fraunhofer Institute IPMS, $\rho_{\mathrm{Si}} \sim 0.001 \Omega \cdot \mathrm{cm}, t_{\mathrm{ox}}=270 \mathrm{~nm}$ ) at 4000RPM and post-anneal at $290{ }^{\circ} \mathrm{C}$ resulting film thickness of $100 \mathrm{~nm}$. We noticed that lower 
concentration of $\mathrm{BCB}$ solution or higher rotation speed for spin-coating sometimes results in inhomogeneous films and thicker BCB film $(>100 \mathrm{~nm})$ would require higher gate voltage range for manipulation because of the lowering of capacitance of the total dielectric. For InSe phototransistors and asymmetric Schottky junctions, few-layer InSe (10-15 nm thick) were mechanically exfoliated from commercially available InSe crystals (HQ Graphene, Netherland) using the scotch tape method and transferred on $\mathrm{SiO}_{2}$ or $\mathrm{BCB}$ in a $\mathrm{N}_{2}$-filled glovebox. Their thickness was monitored by optical microscope combined with Raman spectroscopy and Atomic Force Microscopy (AFM). For BP-InSe heterostructures, few-layer BP flakes (5-10 nm thick) were first mechanically exfoliated onto BCB substrate in glovebox. Few-layer InSe (10-20 nm thick) were immediately transferred onto BP by PDMS to form van der Waals heterostructure. The samples were thermally annealed at $100{ }^{\circ} \mathrm{C}$ inside a vacuum chamber to desorb atmospheric adsorbates and to reinforce the van der Waals contact. For molecular functionalization, didodecyldimethylammonium bromide (DDAB) solution (Tokyo Chemical Industry Co., Ltd (TCI)) with $10^{-6}, 10^{-5}, 10^{-4}, 10^{-3}, 10^{-2}, 10^{-1}$, and $1 \mathrm{~g} / \mathrm{L}$ of concentration were spin-coated at $2000 \mathrm{RPM}$ onto InSe. A post thermal annealing at $120^{\circ} \mathrm{C}$ is applied on hotplate in $\mathrm{N}_{2}$-filled glovebox.

Device fabrication. InSe devices were patterned by photolithography (AZ1505 photoresist and MIF726 developer, Micro Chemicals) using laser writer LW405B from Microtech. For InSe photoFET and BP/InSe P-N photodetector, were thermally evaporated with Plassys MEB 300 following a lift-off process in warm acetone to obtain the final source and drain electrodes. For asymmetric Schottky junctions, one electrode was first patterned with laser writer and $60 \mathrm{~nm}$ Pd was deposited with Egun evaporator Plassys ME300, after lift-off in warm acetone, a second electrode was patterned followed by thermal deposition of $5 \mathrm{~nm}$ chromium and $40 \mathrm{~nm}$ of gold by Plassys MEB300B equipped in the glovebox. The devices were rinsed with acetone and 2-propanol to remove resist residues. All devices were annealed under vacuum at $100^{\circ} \mathrm{C}$ to remove absorbents.

Electrical characterizations. The characterization of device performance was realized by Keithley 2636A under $\mathrm{N}_{2}$ atmosphere. For optoelectronic characterizations, a Polychrome V 
system (Thermo Fisher) was used as monochromatic light source. The output power has been calibrated by a PM100A Power Meter (Thorlabs). The detailed calculation of optoelectronic parameters could be found in Supporting note 1 in Supporting Information.

Raman spectroscopy. Raman and Photoluminescence spectra were carried out in inert atmosphere $\left(\mathrm{N}_{2}\right)$ by Renishaw inVia spectrometer equipped with $532 \mathrm{~nm}$ laser in a nitrogen-filled sealed holder (Linkam). Samples were mounted in the glovebox and immediately measured after annealing or after molecule deposition to avoid exposure to contaminant chemicals. The excitation power was kept below $1 \mathrm{~mW}$ to avoid local heating damage effects. The wavenumber (energy) resolution was $\sim 1 \mathrm{meV}$.

AFM measurement. AFM imaging was performed by means of a Bruker Dimension Icon set-up operating in air, in tapping mode, by using tip model TESPA-V2 (tip stiffness: $k=42 \mathrm{~N} / \mathrm{m}$ ).

KPFM measurement. A Bruker Icon AFM was employed for KPFM experiments. Topography and surface potential (or contact potential difference) images were simultaneously collected with $\mathrm{Pt} / \mathrm{Ir}$ coated silicon probes (Bruker SCM-PIT-V2, resonant frequency $\approx 75 \mathrm{kHz}, \mathrm{k} \approx 3 \mathrm{~N} \bullet$ $\mathrm{m}-1)$ at ambient conditions in the amplitude modulation mode. Macroscopic Kelvin Probe (KP) measurements were performed by using a 2-mm-diameter gold tip amplifier (Ambient Kelvin Probe Package from KP Technology Ltd) at ambient conditions. The calibration of the probe was performed against a freshly cleaved HOPG surface $(4.475 \mathrm{eV}){ }^{[49]}$

XPS measurements: XPS analyses were carried out with a Thermo Scientific K-Alpha X-ray photoelectron spectrometer with a basic chamber pressure of $\sim 10^{-9}$ mbar and an $\mathrm{Al}$ anode as the X-ray source (x-ray radiation of $1486 \mathrm{eV}$ ). Spot sizes of $400 \mu \mathrm{m}$ and pass energies of $200.00 \mathrm{eV}$ for wide energy scans and 10.00-20.00 eV for scans were used.

Computational details. All DFT calculations were performed using the VASP $\operatorname{code}^{55}$ and the projector-augmented wave (PAW) basis set. Exchange and correlation effects are treated at the Perdew-Burke-Ernzerhof (PBE) level with the dispersion forces treated by Grimme 
correction $(\mathrm{PBE}+\mathrm{D} 2)$, with a kinetic energy cut-off of $500 \mathrm{eV}$ and using a Monkhorst-Pack sampling of $3 \times 3 \times 1$ for the Brillouin zone (BZ) integration on the unit cell replicated $4 \times 4 \times 1$ times with the vacuum spacing set to be $40 \AA$ to avoid the interaction with periodic images. ${ }^{[50-53]}$ One Se atom was removed from InSe unit-cell replicated to $4 \times 4 \times 1$ super-cell, generating an optimal vacancy concentration of $\approx 3 \%$, in line with previous investigations ${ }^{[54-56]}$. Dipole moment correction was employed along the 'c' axis ( $Z$ direction \& perpendicular to the InSe surface). Geometries of pristine and defective (-3\% Se vacancies) InSe surfaces, as well as the DDAB adsorbed heterostructures, were fully optimized at the PBE/GGA level of theory prior to the calculation of work function. DDAB molecules were considered with two butyl $\left(\mathrm{C}_{4}\right)$ linkers (dibutyl-dimethyl-ammonium, bromide) instead of two decyl $\left(\mathrm{C}_{10}\right)$ linkers connecting the central nitrogen atom, so as to limit the computational cost. The work function $(\varphi)$ of all the systems was calculated as difference of Fermi energy $\left(E_{f}\right.$, taken as the middle of the band gap) and the electrostatic potential at vacuum level $\left(\mathrm{E}_{\mathrm{p}}\right)$ at the hybrid (HSE06) level of theory.

\section{Data availability}

The data that support the findings of this study are available from the corresponding authors upon reasonable request. Source data are provided with this paper.

\section{REFERENCES}

[1] M. Long, P. Wang, H. Fang, W. Hu, Adv. Funct. Mater. 2019, 29, 1803807.

[2] L. Sang, M. Liao, M. Sumiya, Sensors 2013, 13, 10482.

[3] D. Natali, M. Caironi, in Photodetectors, Elsevier, 2016, 195.

[4] Y. L. Wu, K. Fukuda, T. Yokota, T. Someya, Adv. Mater. 2019, 31, 1903687.

[5] Z. Chen, J. Biscaras, A. Shukla, Nanoscale 2015, 7, 5981.

[6] M. Li, C. Y. Lin, S. H. Yang, Y. M. Chang, J. K. Chang, F. S. Yang, C. Zhong, W. B. Jian, C. H. Lien, C. H. Ho, Adv. Mater. 2018, 30, 1803690.

[7] Y. Yang, J. Jeon, J.-H. Park, M. S. Jeong, B. H. Lee, E. Hwang, S. Lee, ACS Nano 2019, 13, 8804.

[8] W. Feng, J.-B. Wu, X. Li, W. Zheng, X. Zhou, K. Xiao, W. Cao, B. Yang, J.-C. Idrobo, L. Basile, J. Mater. Chem. C 2015, 3, 7022.

[9] M. Long, E. Liu, P. Wang, A. Gao, H. Xia, W. Luo, B. Wang, J. Zeng, Y. Fu, K. Xu, Nano Lett. 2016, $16,2254$.

[10] J. Heo, H. Jeong, Y. Cho, J. Lee, K. Lee, S. Nam, E.-K. Lee, S. Lee, H. Lee, S. Hwang, Nano Lett. 2016, 16, 6746.

[11] Q. Lv, F. Yan, X. Wei, K. Wang, Adv. Opt. Mater. 2018, 6, 1700490.

[12] X. Zhou, X. Hu, B. Jin, J. Yu, K. Liu, H. Li, T. Zhai, Adv. Sci. 2018, 5, 1800478.

[13] O. Lopez-Sanchez, D. Lembke, M. Kayci, A. Radenovic, A. Kis, Nat. Nanotechnol. 2013, 8, 497. 
[14] D. A. Nguyen, H. M. Oh, N. T. Duong, S. Bang, S. J. Yoon, M. S. Jeong, ACS Appl. Mater. Interfaces 2018, 10, 10322.

[15] W. Zhang, M.-H. Chiu, C.-H. Chen, W. Chen, L.-J. Li, A. T. S. Wee, ACS Nano 2014, 8, 8653.

[16] S. R. Tamalampudi, Y.-Y. Lu, R. K. U, R. Sankar, C.-D. Liao, C.-H. Cheng, F. C. Chou, Y.-T. Chen, Nano Lett. 2014, 14, 2800.

[17] D. A. Bandurin, A. V. Tyurnina, L. Y. Geliang, A. Mishchenko, V. Zólyomi, S. V. Morozov, R. K. Kumar, R. V. Gorbachev, Z. R. Kudrynskyi, S. Pezzini, Nat. Nanotechnol. 2017, 12, 223.

[18] M. Gobbi, E. Orgiu, P. Samorì, Adv. Mater. 2018, 30, 1706103.

[19] S. Bertolazzi, M. Gobbi, Y. Zhao, C. Backes, P. Samorì, Chem. Soc. Rev. 2018, 47, 6845.

[20] B. Chamlagain, Q. Li, N. J. Ghimire, H. J. Chuang, M. M. Perera, H. Tu, Y. Xu, M. Pan, D. Xaio, J. Yan, D. Mandrus, Z. Zhou, ACS Nano 2014, 8, 5079.

[21] K. Dolui, I. Rungger, S. Sanvito, Phys. Rev. B 2013, 87, 165402.

[22] Y. Zhao, S. Bertolazzi, P. Samorì, ACS Nano 2019, 13, 4814.

[23] Z. Yu, Z. Y. Ong, S. Li, J. B. Xu, G. Zhang, Y. W. Zhang, Y. Shi, X. Wang, Adv. Funct. Mater. 2017, 27, 1604093.

[24] A. H. Edwards, in The Physics and Chemistry of $\mathrm{SiO}_{2}$ and the $\mathrm{Si}_{-} \mathrm{SiO}_{2}$ Interface, Springer, 1988, 271.

[25] E. H. Poindexter, P. J. Caplan, G. J. Gerardi, in The Physics and Chemistry of $\mathrm{SiO}_{2}$ and the Si-SiO Interface, Springer, 1988, 299.

[26] Y. Guo, X. Wei, J. Shu, B. Liu, J. Yin, C. Guan, Y. Han, S. Gao, Q. Chen, Appl. Phys. Lett. 2015, 106, 103109.

[27] X. Dong, D. Fu, W. Fang, Y. Shi, P. Chen, L. J. Li, Small 2009, 5, 1422.

[28] Y. C. Du, H. Liu, A. T. Neal, M. W. Si, P. D. Ye, IEEE Electron Device Lett. 2013, 34, 1328.

[29] M.-A. Stoeckel, M. Gobbi, T. Leydecker, Y. Wang, M. Eredia, S. Bonacchi, R. Verucchi, M. Timpel, M. V. Nardi, E. Orgiu, ACS Nano 2019, 13, 11613.

[30] Y. Wang, A. Slassi, M.-A. Stoeckel, S. Bertolazzi, J. Cornil, D. Beljonne, P. Samorì, J. Phys. Chem. Lett. 2019, 10, 540.

[31] Y. Wang, S. M. Gali, A. Slassi, D. Beljonne, P. Samorì, Adv. Funct. Mater. 2020, 30, 2002846.

[32] J. Sun, Y. Wang, S. Guo, B. Wan, L. Dong, Y. Gu, C. Song, C. Pan, Q. Zhang, L. Gu, Adv. Mater. 2020, 32, 1906499.

[33] S. Mouri, Y. Miyauchi, K. Matsuda, Nano Lett. 2013, 13, 5944.

[34] W. Luo, Y. Cao, P. Hu, K. Cai, Q. Feng, F. Yan, T. Yan, X. Zhang, K. Wang, Adv. Opt. Mater. 2015, 3, 1418.

[35] Q. Zhao, W. Wang, F. Carrascoso-Plana, W. Jie, T. Wang, A. Castellanos-Gomez, R. Frisenda, Mater. Horiz. 2020, 7, 252.

[36] M. S. Choi, D. Qu, D. Lee, X. Liu, K. Watanabe, T. Taniguchi, W. J. Yoo, ACS Nano 2014, 8, 9332.

[37] J. S. Ross, P. Klement, A. M. Jones, N. J. Ghimire, J. Yan, D. Mandrus, T. Taniguchi, K. Watanabe, K. Kitamura, W. Yao, Nat. Nanotechnol. 2014, 9, 268.

[38] M. Dai, H. Chen, F. Wang, M. Long, H. Shang, Y. Hu, W. Li, C. Ge, J. Zhang, T. Zhai, ACS Nano 2020, 14, 9098.

[39] M. Dai, H. Chen, R. Feng, W. Feng, Y. Hu, H. Yang, G. Liu, X. Chen, J. Zhang, C.-Y. Xu, ACS Nano 2018, 12, 8739.

[40] S. Hu, Q. Zhang, X. Luo, X. Zhang, T. Wang, Y. Cheng, W. Jie, J. Zhao, T. Mei, X. Gan, Nanoscale 2020, 12, 4094.

[41] D. J. Perello, S. H. Chae, S. Song, Y. H. Lee, Nat. Comm. 2015, 6, 1.

[42] C. Kim, I. Moon, D. Lee, M. S. Choi, F. Ahmed, S. Nam, Y. Cho, H.-J. Shin, S. Park, W. J. Yoo, ACS Nano 2017, 11, 1588.

[43] Y.-H. Chen, C.-Y. Cheng, S.-Y. Chen, J. S. D. Rodriguez, H.-T. Liao, K. Watanabe, T. Taniguchi, C.-W. Chen, R. Sankar, F.-C. Chou, NPJ 2D Mater. Appl. 2019, 3, 1.

[44] R. Cao, H. D. Wang, Z. N. Guo, D. K. Sang, L. Y. Zhang, Q. L. Xiao, Y. P. Zhang, D. Y. Fan, J. Q. Li, H. Zhang, Adv. Opt. Mater. 2019, 7, 1900020.

[45] S. Zhao, J. Wu, K. Jin, H. Ding, T. Li, C. Wu, N. Pan, X. Wang, Adv. Funct. Mater. 2018, 28, 1802011.

[46] Y. Deng, Z. Luo, N. J. Conrad, H. Liu, Y. Gong, S. Najmaei, P. M. Ajayan, J. Lou, X. Xu, P. D. Ye, ACS Nano 2014, 8, 8292.

[47] S. Cao, Y. Xing, J. Han, X. Luo, W. Lv, W. Lv, B. Zhang, Z. Zeng, Nanoscale 2018, 10, 16805.

[48] X. K. Li, X. G. Gao, B. W. Su, W. Xin, K. X. Huang, X. Q. Jiang, Z. B. Liu, J. G. Tian, Adv. Mater. Interfaces 2018, 5, 1800960. 
[49] W. N. Hansen, G. J. Hansen, Surf. Sci. 2001, 481, 172.

[50] G. Kresse, J. Furthmüller, Phys. Rev. B 1996, 54, 11169.

[51] G. Kresse, D. Joubert, Phys. Rev. B 1999, 59, 1758.

[52] J. P. Perdew, K. Burke, M. Ernzerhof, Phys. Rev. Lett. 1996, 77, 3865.

[53] S. Grimme, J. Comput. Chem. 2006, 27, 1787.

[54] D. Chen, X. Zhang, H. Cui, J. Tang, S. Pi, Z. Cui, Y. Li, Y. Zhang, Appl. Surf. Sci. 2019, 479, 852.

[55] X. Liu, J.-C. Ren, S. Zhang, M. Fuentes-Cabrera, S. Li, W. Liu, J. Phys. Chem. Lett. 2018, 9, 3897.

[56] B. Ma, Y. Peng, D. Ma, Z. Deng, Z. Lu, Appl. Surf. Sci. 2019, 495, 143463.

\section{ACKNOWLEDGEMENTS}

We thank Dr. Yuda Zhao (Zhejiang University, China) and Dr. Chun Ma (University of Strasbourg, France) for fruitful discussions. We acknowledge funding from European Commission through the ERC project SUPRA2DMAT (GA-833707), the Graphene Flagship Core 3 project (GA- 881603) and the Marie Curie ITN project UHMob (GA-811284), the Agence Nationale de la Recherche through the Labex projects CSC (ANR-10-LABX-0026 CSC) and NIE (ANR-11-LABX-0058 NIE) within the Investissement d'Avenir program (ANR-10-120 IDEX-0002-02), the International Center for Frontier Research in Chemistry (icFRC). The work in Mons is supported by the Belgian National Fund for Scientific Research (FRS-FNRS), within FNRS-PDR-TOREADOR project. Computational resources were provided by the Consortium des Équipements de Calcul Intensif (CÉCI) funded by F.R.S.-FNRS under Grant 2.5020.11. DB is FNRS Research Director.

\section{AUTHOR INFORMATION}

PS, YW and HW conceived and coordinated the work. YW worked on sample preparation, device fabrication, optical and electrical characterization. HW worked on the molecule selection and preparation. SMG did the modelling work, under the supervision of DB. NT performed the KPFM. YW measured the AFM with CW. YY and YW established the optoelectronic measurement setup. YC participated in metal evaporation. YW analysed the data. YW and PS wrote the paper with all the authors contributing to the discussion and preparation of the manuscript. 
Competing interests: The authors declare no competing interests.

\section{Additional information}

Correspondence and requests for materials: should be addressed to P.S. 

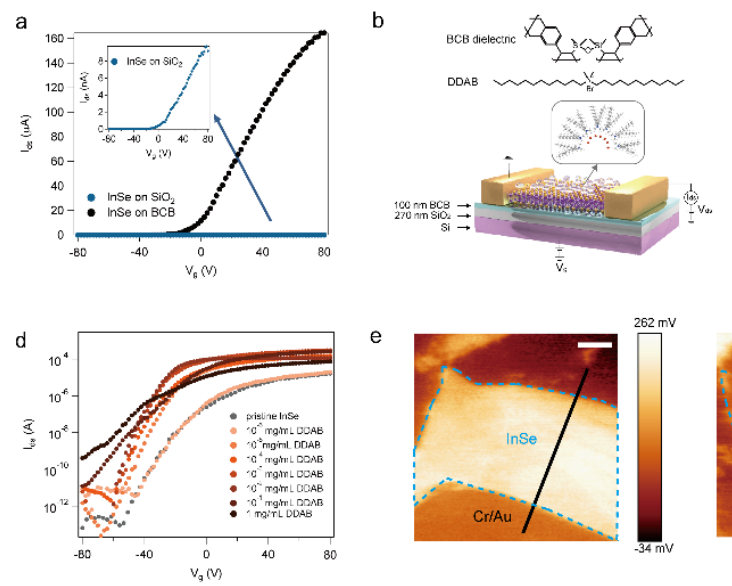

e
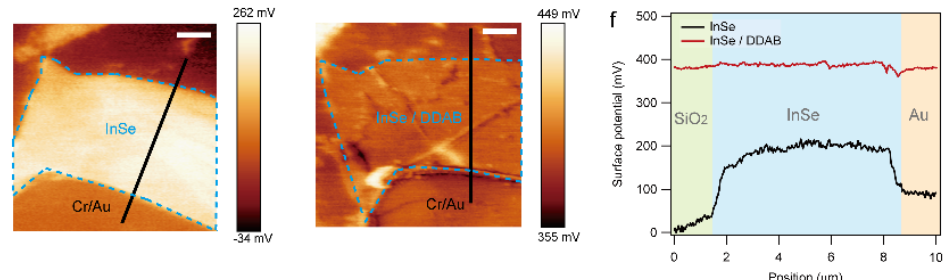

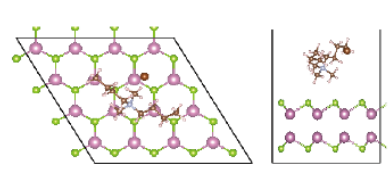

Molecular Conformation 1

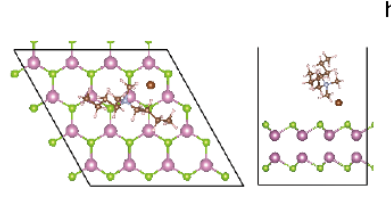

Molecular Conformation 2

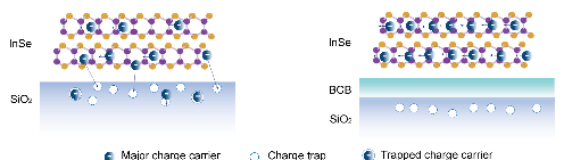

Figure 1. Dielectric engineering and molecular doping of InSe FET. a) Comparison of transfer $\left(\mathrm{V}_{\mathrm{g}}-\mathrm{I}_{\mathrm{ds}}\right)$ curve of InSe FET onto $\mathrm{SiO}_{2}$ and $\mathrm{BCB}$. The inset shows the zoom of transfer curve on $\mathrm{SiO}_{2}$. b) Illustration of device structure and the chemical structure of BCB polymer and DDAB molecule used in this work. c) Scheme of the mechanism of charge transport enhancement of InSe on $\mathrm{SiO}_{2}$ and $\mathrm{BCB}$. d) Transfer $\left(\mathrm{V}_{\mathrm{g}}-\mathrm{I}_{\mathrm{ds}}\right)$ curve of InSe FET functionalized with DDAB with different concentration. e,f ) Surface potential measured by Kelvin probe force microscopy (KPFM) of few-layer InSe of before and after functionalization with DDAB (scale bar: $3 \mu \mathrm{m}$ ) and (f) the corresponding line potential profile of marked in black line in (e). The flake is grounded by a $\mathrm{Cr} / \mathrm{Au}$ electrode during the measurement $\mathrm{g}$ ) Schematic representation of DDAB physisorbed onto InSe in conformation 1 and conformation $2 . \mathrm{h}$ ) Summary of DFT calculated work function, Bader charge transfer, molecular dipole moment and adsorption energy of InSe and defective InSe functionalized with DDAB. The smaller work function shift of the experimental value is attributed to the inevitable p-doping to InSe from the water and oxygen in air during the measurement. 

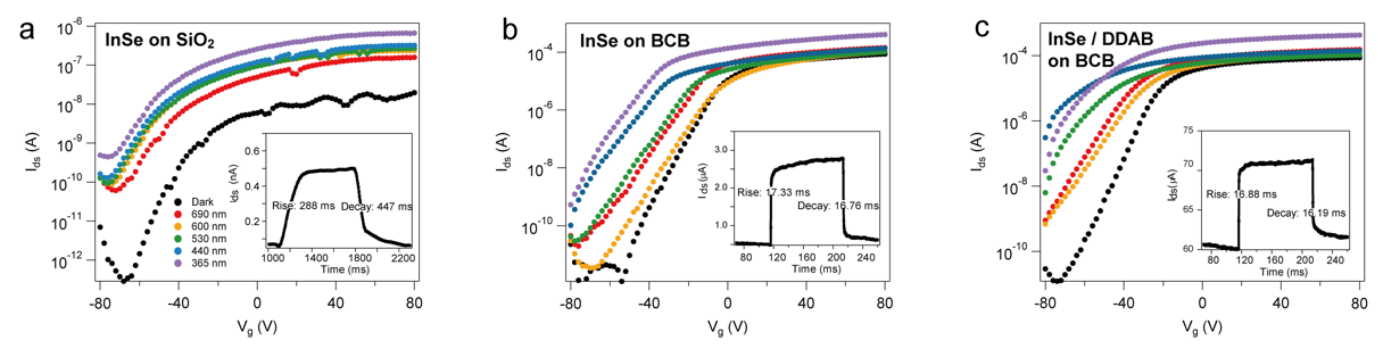

d
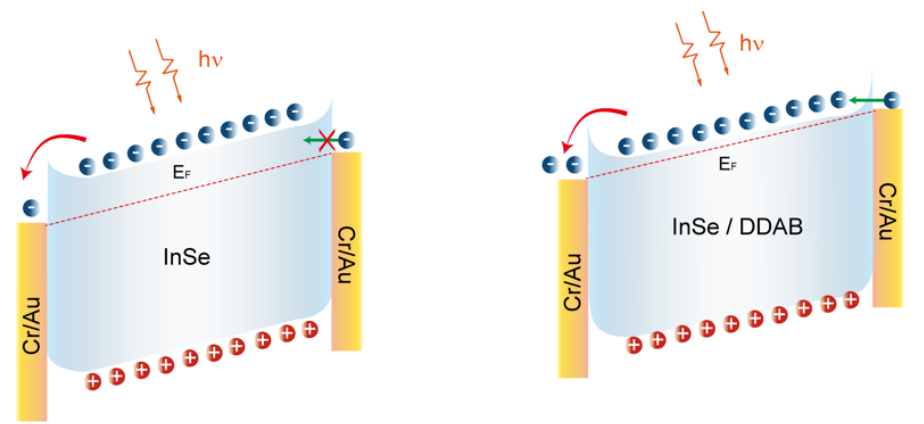

$\mathrm{V}_{\text {th }}>0 \mathrm{~V}$

$\mathrm{V}_{\text {th }}<0 \mathrm{~V}$
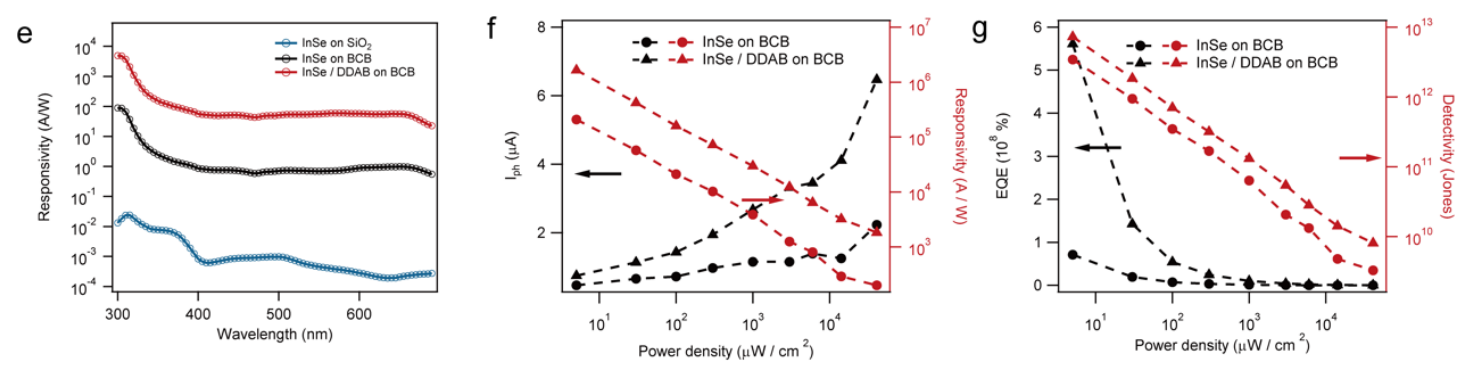

Figure 2. Photoresponsive measurements of photoFET. a,b,c) $\mathrm{Ids}_{\mathrm{ds}}-\mathrm{V}_{\mathrm{g}}$ curve of (a) $\mathrm{InSe}$ on $\mathrm{SiO}_{2}$, (b) InSe on $\mathrm{BCB}$ and (c) InSe/DDAB on $\mathrm{BCB}$ at $\mathrm{V}_{\mathrm{g}}=0 \mathrm{~V}$ and $\mathrm{V}_{\mathrm{ds}}=1 \mathrm{~V}$. Inset: time-resolved photoresponse under the illumination of $365 \mathrm{~nm}$ at $34.3 \mathrm{~mW} / \mathrm{cm}^{2}$. d) Band diagram of InSe photoFET before and after functionalization with $\mathrm{DDAB}$ at $\mathrm{V}_{\mathrm{g}}=0 \mathrm{~V}$. The power density is adjusted to $34.3 \mathrm{~mW} / \mathrm{cm}^{2}$. e) Calculated responsivity with of wavelength scan from $300 \mathrm{~nm}$ to $690 \mathrm{~nm}$ at high incident light power (34.3 $\mathrm{mW} / \mathrm{cm}^{2}$ for $365 \mathrm{~nm}$ light). The interval of wavelength is $5 \mathrm{~nm}$. f,g) Power dependence of (f) photocurrent and responsivity and (g) EQE and detectivity of $\mathrm{InSe}$ on $\mathrm{SiO}_{2}$, InSe on $\mathrm{BCB}$ and InSe $/ \mathrm{DDAB}$ on $\mathrm{BCB}$ at $\mathrm{V}_{\mathrm{g}}=0 \mathrm{~V}$ and $\mathrm{V}_{\mathrm{ds}}=1 \mathrm{~V}$ illuminated with $365 \mathrm{~nm}$ light. 


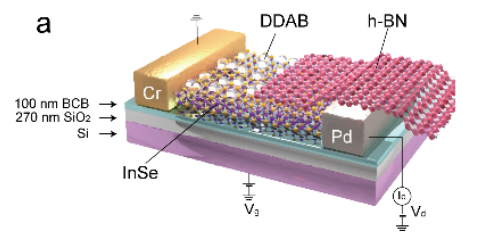

b

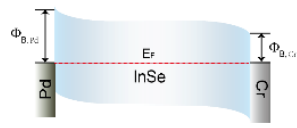

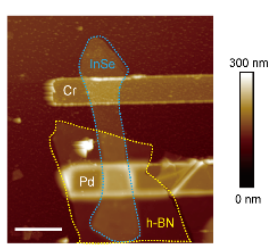
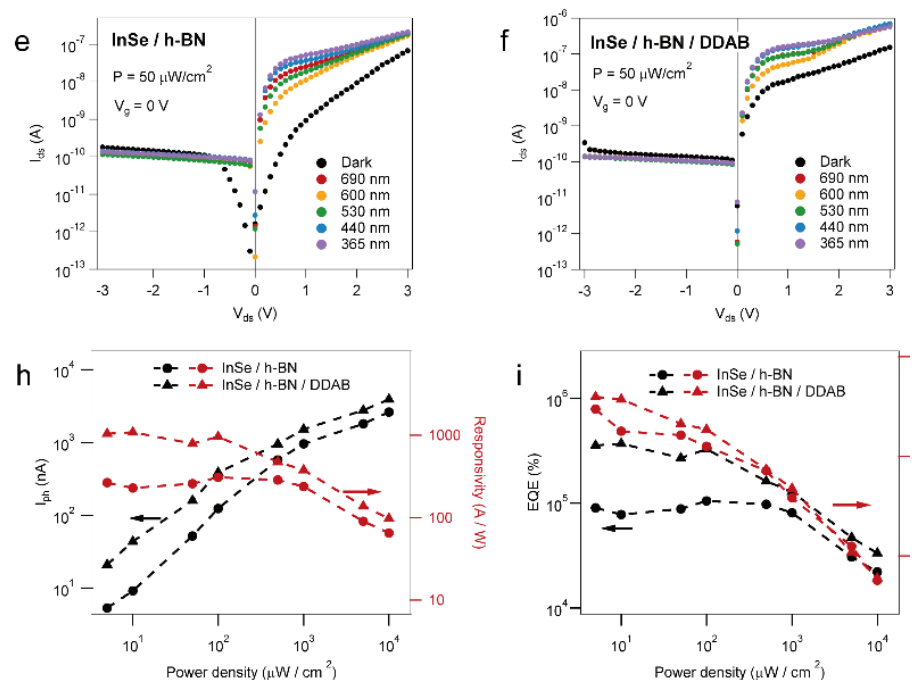

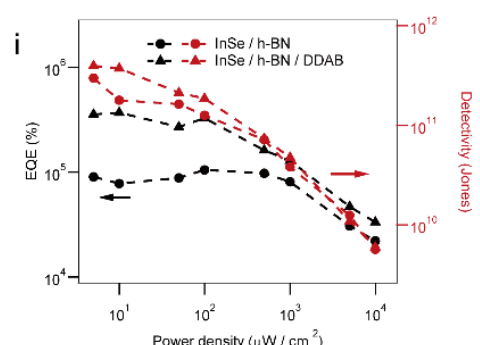

Figure 3. Molecular functionalization of lateral InSe asymmetric Schottky junction. a) Illustration of device structure of lateral InSe asymmetric Schottky junction. b) Band alignment of InSe with Pd and Cr contacts. Here, the Schottky barrier height (SBH) was estimated from Ref. ${ }^{[43]}$ where the SBH is 280 $\mathrm{meV}$ and $560 \mathrm{meV}$ for $\mathrm{Cr}$ and Pd respectively. c) AFM image of representative lateral InSe asymmetric Schottky junction partially covered with few-layer h-BN on top. The scale bar is $6 \mu \mathrm{m}$. d) Gate-dependent I-V curves of InSe/h-BN before and after doping with DDAB in dark condition. e) Photodetection of lateral InSe asymmetric Schottky junction before doping with DDAB. f) Photodetection of lateral InSe asymmetric Schottky junction after doping with DDAB. The weak photoresponse at reverse bias indicate a back-to-back connected diode with only one side illuminated due to the presence of Schottky barriers at both contacts. g) Time-dependent photoresponse of power density ranging from $5 \mu \mathrm{W} / \mathrm{cm}^{2}$ to $5000 \mu \mathrm{W} / \mathrm{cm}^{2}$ of InSe/h-BN and InSe/h-BN/DDAB. h,i) Power dependence of (h) photocurrent and responsivity and (i) EQE and $D^{*}$ of InSe/h-BN InSe/h-BN/DDAB on $\mathrm{BCB}$ at $\mathrm{V}_{\mathrm{g}}=0 \mathrm{~V}$ and $\mathrm{V}_{\mathrm{d}}=1 \mathrm{~V}$ illuminated with $365 \mathrm{~nm}$ light. 
a
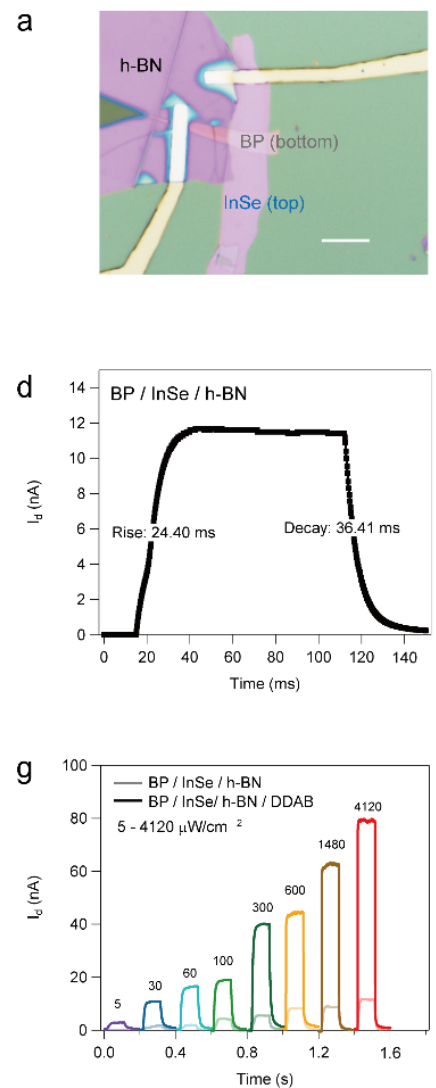
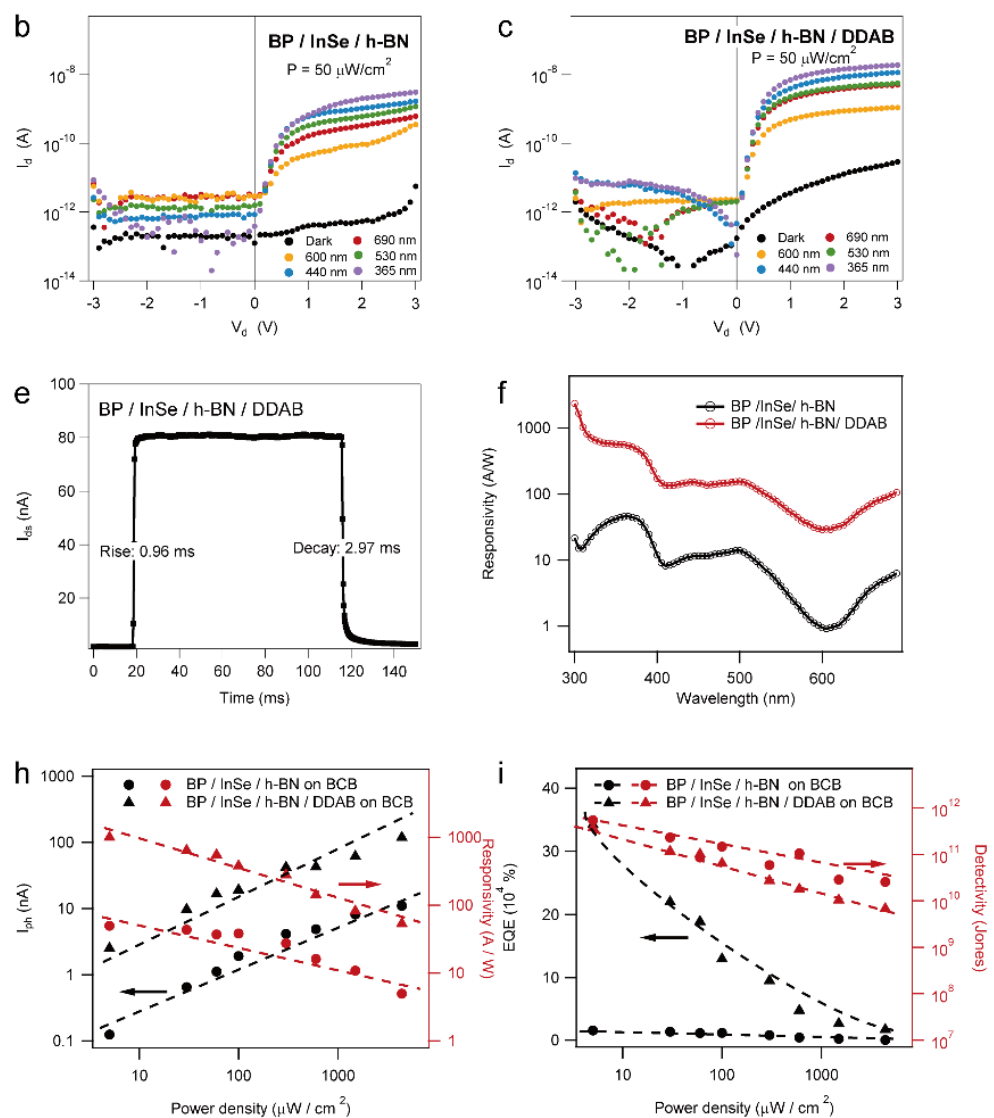

Figure 4. Molecular functionalization of BP-InSe P-N junctions. a) Optical image of BP/InSe heterostructure partially encapsulated by h-BN. b,c) Output curves of BP/InSe/h-BN heterostructure (b) before and (c) after the functionalization of DDAB. On BCB substrate, due to the enhanced n-type transport also in $\mathrm{BP}$ (as it is ambipolar under $\mathrm{Cr}$ contact), we have observed larger photoresponse at forward bias than reverse bias. d,e) Time-resolved photoresponse of (d) BP/ InSe/ h-BN and (e) BP/ InSe/ h-BN/ DDAB at $V_{\mathrm{g}}=0 \mathrm{~V}$ and $\mathrm{V}_{\mathrm{d}}=1 \mathrm{~V}$ under the illumination of $365 \mathrm{~nm}$. Light power is adjusted at $4.12 \mathrm{~mW} / \mathrm{cm}^{2}$. f) Spectral responsivity of wavelength scan from $300 \mathrm{~nm}$ to $690 \mathrm{~nm}$ at $\mathrm{V}_{\mathrm{g}}=0 \mathrm{~V}$ and $\mathrm{V}_{\mathrm{ds}}=1 \mathrm{~V}$. The interval of wavelength is $5 \mathrm{~nm}$. g) Photoresponse of power density ranging from $5 \mu \mathrm{W} / \mathrm{cm}^{2}$ to $4120 \mu \mathrm{W} / \mathrm{cm}^{2}$ of BP/ InSe/h-BN and BP/InSe/h-BN/DDAB. h,i) Power dependence of (h) photocurrent and responsivity and (i) EQE and $D^{*}$ of $\mathrm{BP} / \mathrm{InSe} / \mathrm{h}-\mathrm{BN}$ and $\mathrm{InSe} / \mathrm{h}-\mathrm{BN} / \mathrm{DDAB}$ at $\mathrm{V}_{\mathrm{g}}=\mathrm{V}$ and $V_{\mathrm{d}}=1 \mathrm{~V}$ illuminated with $365 \mathrm{~nm}$ light. 